\title{
A Distinct Human Papilloma Virus Epidemiological Profile of Korean-Chinese Women Living in Yanbian, China
}

\author{
Qunying Wu $\mathbf{u}^{1,2}$, Jin-Ho Kuk ${ }^{1}$, Young-Joon Ryu ${ }^{1}$ \\ ${ }^{1}$ Department of Pathology, College of Medicine, Kangwon National University, Chun-Cheon, Republic of Korea \\ ${ }^{2}$ Department of Pathology, Yanbian Maternal and Child Health Care Hospital, Yanji, P. R. China
}

\begin{abstract}
Summary
Cervical cancer is a common in women worldwide. Human papilloma virus (HPV), which is known to be a direct cause of cervical cancer, differs in its infection rate and genotype by country, geography, race, genetic factors, and immune status. No previously reported studies have reported the HPV prevalence and genotype distributions of Korean-Chinese women living in China. This study examined the prevalence and genotype distribution of HPV among Korean-Chinese women to find similarities and differences compared to HanChinese women and Korean women. We also evaluated the effectiveness of a genotyping test compared with liquid-based cytology (LBC) as a screening test. We performed LBC and HPV genotyping tests on 2099 women living in the Yanbian region. The mean age of the 2099 subjects was 42.5 years (18-82). The largest ethnic groups were Han-Chinese (78\%), Korean-Chinese (20.2\%), Man-Chinese $(0.8 \%)$, Jang-Chinese $(0.04 \%)$, and Myo-Chinese $(0.04 \%)$. Of the Korean-Chinese women, $34.4 \%$ tested positive for HPV; the most prevalent subtypes were 52, 58, 16, 53, and 33, which was clearly different from those of Han-Chinese women and other Koreans. The results of LBC showed a false negative rate of $62.4 \%$ compared with the results of HPV genotyping tests. This shows that a cervical cancer screening system utilizing HPV genotyping or a combined method is more effective. According to the HPV profile, only the Gardasil 9 vaccine can cover the HPV subtypes prevalent in Korean-Chinese women in the Yanbian region. This paper is the first report on HPV epidemiology among Korean-Chinese women in China.
\end{abstract}

Key words: Human papilloma virus; Uterine cervical neoplasm; HPV genotyping; Vaccine.

\section{Introduction}

Uterine cervical cancer is a common malignant tumor in women worldwide and it is one of the leading causes of death from cancer [1]. Infection with human papilloma virus (HPV) is the main cause of invasive cervical cancer or progenitor lesions, which are observed in $90 \%$ of patients with cervical cancer [2]. Infection with HPV increases the risk of future epithelial lesions even if there is no histological change in the cervix. The link between HPV infection and cervical cancer has been reported in many studies [38]. Thus far, more than 100 genotypes of HPV have been described, of which about 40 cause infections in the genitals $[9,10]$. HPV is divided into high-risk and low-risk groups according to how the virus affects progression to malignant tumors. High-risk HPV strains (16, 18, 31, 33, $35,39,45,51,52,53,56,58,59,66,68$, etc.) are strongly associated with cancer [9-14]. In particular, high-risk HPV subtypes such as HPV 16, 18, and 31 have been shown to have biochemical properties that can cause invasive cervical cancer [15-17]. Low-risk HPV genotypes such as HPV $6,11,42$, and 43 are associated with benign lesions such as condyloma and warts $[9,10]$. The worldwide distribution of HPV genotypes is very regional $[18,19]$. HPV infection is affected by geographic location, race, immunological status, and genetic factors [20-22]. In molecular analyses of women without cytological abnormalities, HPV infection rates were higher in less developed countries $(15.5 \%)$ than in more developed countries $(10.0 \%)$, and in older women compared to young women [18]. In most cases, HPV 16 and 18 are the most common genotypes associated with invasive epithelial cell carcinoma, followed by HPV 45, 31, and 33. In Asian women, however, HPV 58 and 52 are more common than HPV 45, 31, and 33 [23, 24]. There are two methods of HPV detection that are widely used in the clinic, namely mixed capture technology and polymerase chain reaction (PCR). Currently, Chinese medical institutions use the $23 \mathrm{HPV}$ Genotyping Real-time PCR Kit (Hybribio, HBRT-23) for the diagnosis of individuals and to collect information regarding large-scale HPV trends and distribution. It is an approved fluorescence-based PCR technology, which tests for 23 HPV subtypes $(16,18,31$, $33,35,39,45,51,52,53,56,58,59,66,68,6,11,42,43$, $44,81,73,82$, and 23 ). The two most common vaccines that have been developed to prevent HPV subtypes that can cause cervical cancer are Gardasil (Merck, Sharp \& Dohme Corp., New Jersey, US) and Cervarix (GlaxoSmithKline Biologicals s.a., Rixensart, Belgium) [25]. The Cervarix $2 \mathrm{HPV}$ vaccine is used to prevent infection by HPV 16 and 18. The Gardasil 4 vaccine can prevent infection by HPV $6,11,16$ and 18 , and the Gardasil 9 vaccine can prevent infection by HPV 6, 11, 16, 18, 31, 33, 45, 52, and 58 [26].

In this study, the molecular genotype results were com-
Eur. J. Gynaecol. Oncol. - ISSN: 0392-2936 XLI, n. 5, 2020

doi: 10.31083/j.ejgo.2020.05.c5439
This is an open access article under the CC BY-NC 4.0 license (https://creativecommons.org/licenses/by-nc/4.0/). 
pared to the liquid-based cytology (LBC) screening results of Korean-Chinese women living in the Yanbian Korean Autonomous Region, Jilin Province, China. Our aim was to determine the most appropriate vaccine for HPV prevention in this area.

\section{Patients and Methods}

\section{Patients}

The current study included 2099 patients who visited the Department of Obstetrics and Gynecology at Yanbian Maternal and Child Health Care Hospital in Jilin Province, China, from April 2018 to November 2018. The experimental plan was conducted with the approval of the Institutional Ethics Committee of Kangwon National University and the experiments were conducted in China.

\section{Liquid-based cytology and HPV genotype tests}

All 2099 specimens underwent cervical fluid cytology E-prep 1000 (Jilin Shiye Medical Technology Co. Ltd., China) and HPV DNA Test (Hybribio Limited, Chauzou, China) at the same time. Tissue sampling and slide preparation was performed according to the protocol provided with the E-Prep 1000 Test. The prepared slides were first assessed by a pathologist and then reassessed by another pathologist. All abnormal readings were reassessed, and the diagnosis was confirmed by three pathologists working together. Cytological diagnosis was performed using the 2014 Bethesda system and samples were categorized as follows: low-grade squamous intraepithelial lesion (LSIL), high-grade squamous intraepithelial lesion (HSIL), atypical squamous cells of undetermined significance (ASCUS), atypical squamous cells, cannot exclude HSIL (ASC-H), squamous cell carcinoma (SCC), or adenocarcinoma (AC).

HPV genotyping was performed using a 23 HPV Genotyping Real-Time PCR Kit (Hybribio, HBRT-23, Hong Kong). This test can detect 15 high-risk HPVs $(16,18,31$, $33,35,39,45,51,52,53,56,58,59,66,68)$ and 8 low-risk HPVs $(6,11,42,43,44,81,73,82)$, allowing analysis of a total of $23 \mathrm{HPV}$ genotypes. After processing the sample, the extracted DNA was combined with the primer-containing real-time PCR master mix provided in the 23 HPV Genotyping Real-time PCR Kit, and PCR was performed according to the protocol provided by the manufacturer for gene amplification. If the $\mathrm{Ct}$ value of the globulin internal control was below 40 cycles, the $\mathrm{Ct}$ value of the other sequences was not clear and was interpreted as negative. When the sample Ct value was 40-45 cycles, the sample was evaluated again. If the $\mathrm{Ct}$ value of the second test result was less than 40, the sample was considered to be positive for HPV, otherwise it was interpreted as negative. If the $\mathrm{Ct}$ values of the sample were not checked, the results were considered invalid and samples were retaken (Table 1).

\section{Statistical analysis}

The Statistical Package for the Social Sciences (SPSS) software (SPSS for Windows version 12.0, Chicago, IL, USA) was used for statistical analyses.

\section{Results}

\section{Patient characteristics}

The mean age of the 2099 subjects was 42.5 years, with the youngest subject at 18 years old and the oldest at 82 . When grouped by age, the 40-49 years' age group was the largest at $33.2 \%(697 / 2099)$, followed by $30-39$ years at $31.3 \%$ (658/2099), 50-59 years at 19.5\% (409/2099), 20-29 years at $10.1 \%(213 / 2099), 60-69$ years at $4.8 \%(101 / 2099)$, over 70 years old at $0.9 \%(18 / 2099)$, and under 20 years old at $0.14 \%(3 / 2099)$ (Table 2$)$. The largest ethnic group was Han-Chinese, who comprised $78 \%$ of the study population (1656/2099), while Korean-Chinese comprised 20.2\% (424/2099), Man-Chinese, 0.8\% (17/2099), Jang-Chinese, $0.04 \%$ (1/2099), and Myo-Chinese, 0.04\% (1/2099) (Table 3).

\section{Liquid-based cytology test results}

The LBC test was conducted according to the 2014 Bethesda system. When cytological results were calculated as a proportion of the study population and grouped according to age, the abnormal cell frequency was $4.3 \%$ in the 30 39 years' group (88/2099), 3.9\% in the $40-49$ years' group (83/2099), $3.4 \%$ in the 50-59 years group (72/2099), $2.1 \%$ in the 20-29 years group (40/2099), $1.3 \%$ in the $60-69$ years group (27/2099), and $0.3 \%$ in the over 70 years old group (6/2099). Among them, the rankings of medium or highergrade by age were $1 \%$ in the $40 \mathrm{~s}(21 / 2099), 0.88 \%$ in the $30 \mathrm{~s}(18 / 2099), 0.7 \%$ in the $50 \mathrm{~s}$ (14/2099), $0.4 \%$ in the $20 \mathrm{~s}$ and $60 \mathrm{~s}(8 / 2099)$, and $0.08 \%$ in the $70 \mathrm{~s}(2 / 2099)$. Findings within the normal range were observed in 1783 women (85\%), which included normal limits, atrophy, and reactive cellular changes associated with inflammation. Of the 316 women (15\%) who exhibited abnormal findings, $10.1 \%$ (213/2099) were diagnosed with ASCUS, 2.1\% (44/2099) with ASC-H, 1.5\% (32/2099) with LSIL, 1.1\% (23/2099) with HSIL, 0.14\% (3/2099) with SCC, and 0.04\% (1/2099) with AC (Table 2). As a result of cross-analysis to examine differences in test results according to the age group, the chi-squared statistic was 33.673 ( $p$-value $=0.001)$. It can be said that there is a significant difference in the test results depending on the age group $(p<0.05)$. The cytological results of the Korean-Chinese group showed that $83.7 \%$ were diagnosed as normal (355/424) and $16.3 \%$ were abnormal (69/424). Of the abnormal cytology results, $11.8 \%$ were diagnosed as ASCUS (50/424), 2.1\% as ASC-H (9/424), 1.2\% as LSIL (5/424), 0.9\% as HSIL $(4 / 424)$, and $0.2 \%$ as SCC $(1 / 424)$. Of the Han-Chinese test results, $85.4 \%(1414 / 1656)$ were normal and $14.6 \%$ (242/1656) were abnormal. Among the abnormal cytology results, 9.6\% were diagnosed as ASCUS (159/1656), $2.1 \%$ as ASC-H (34/1656), $1.6 \%$ as LSIL (27/1656), $1.1 \%$ as HSIL (19/1656), $0.1 \%$ as SCC (2/1656), and $0.1 \%$ as AC (1/1656). The Man-, Jang-, and Myo-Chinese groups did not yield statistically significant results due to the small number of patients in each group (Table 3 ). As a result of cross-analysis to examine the difference in test results ac- 
Table 1. - Interpretation protocol of real-time PCR results for human papilloma virus genotyping.

\begin{tabular}{lcccc}
\hline PCR reaction tube (HPV subtypes) & \multicolumn{2}{l}{ FAM Ct $\leq$} & \multicolumn{2}{l}{$\begin{array}{l}\text { HEX Ct } \leq \\
40\end{array}$} \\
& 40 & $\begin{array}{l}\text { ROX Ct } \leq \\
40\end{array}$ & CY5 Ct $\leq 40$ \\
\hline PCR Mix 1 (16/18/31/33) & 16 & 18 & 31 & 33 \\
PCR Mix 2 (35/39/45/51) & 35 & 39 & 45 & 51 \\
PCR Mix 3 (52/56/58/59) & 52 & 56 & 58 & 59 \\
PCR Mix 4 (66/68/6/11) & 66 & 68 & 6 & 11 \\
PCR Mix 5 (42/43/44/53) & 42 & 43 & 44 & 53 \\
PCR Mix 6 (81/73/82/IC) & 81 & 73 & 82 & globulin \\
\hline
\end{tabular}

Table 2. - Age distribution and result of liquid-based cytological examination.

\begin{tabular}{lcccccccc}
\hline \multirow{2}{*}{$\begin{array}{l}\text { Age } \\
\text { year) }\end{array}$} & Normal & ASCUS & LSIL & ASC-H & HSIL & SCC & AC & \multirow{2}{*}{ Total } \\
\cline { 2 - 7 }$<20$ & $3(0.14)$ & 0 & 0 & 0 & 0 & 0 & 0 & $3(0.14)$ \\
$20-29$ & $173(8.2)$ & $27(1.3)$ & $5(0.4)$ & $6(0.3)$ & $2(0.1)$ & 0 & 0 & $213(10.1)$ \\
$30-39$ & $570(27.2)$ & $58(2.8)$ & $12(0.6)$ & $14(0.7)$ & $3(0.14)$ & $1(0.04)$ & 0 & $658(31.3)$ \\
$40-49$ & $614(29.3)$ & $56(2.7)$ & $6(0.3)$ & $13(0.6)$ & $8(0.4)$ & 0 & 0 & $697(33.2)$ \\
$50-59$ & $337(16.1)$ & $50(2.4)$ & $8(0.4)$ & $7(0.3)$ & $5(0.4)$ & $1(0.04)$ & $1(0.04)$ & $409(19.5)$ \\
$60-69$ & $74(3.5)$ & $18(0.9)$ & $1(0.04)$ & $4(0.2)$ & $4(0.2)$ & 0 & 0 & $101(4.8)$ \\
$\geq 70$ & $12(0.6)$ & $4(0.2)$ & 0 & 0 & $1(0.04)$ & $1(0.04)$ & 0 & $18(0.9)$ \\
Total (No/\%) & $1783(84.9)$ & $213(10.1)$ & $32(1.5)$ & $44(2.1)$ & $23(1.1)$ & $3(0.14)$ & $1(0.04)$ & $2099(100)$ \\
\hline
\end{tabular}

No: Number of patients; ASCUS: atypical squamous cells of undetermined significance; LSIL: low-grade squamous intraepithelial lesion; ASC-H: atypical squamous cells, cannot exclude HSIL; HSIL: high-grade squamous intraepithelial lesion; SCC: squamous cell carcinoma; AC: adenocarcinoma.

Table 3. - Ethnic distribution of liquid-based cytological result.

\begin{tabular}{lcccccc}
\hline \multirow{2}{*}{$\begin{array}{c}\text { Cytology } \\
\text { diagnosis }\end{array}$} & Han-Chinese & Korean-Chinese & Man-Chinese & Jang-Chinese & Myo-Chinese & Total \\
\cline { 2 - 5 } Normal & $1414(67.3)$ & $355(17.0)$ & $13(0.6)$ & $1(0.04)$ & 0 & $1783(85)$ \\
ASCUS & $159(7.6)$ & $50(2.4)$ & $3(0.14)$ & 0 & $1(0.04)$ & $213(10.1)$ \\
LSIL & $27(1.3)$ & $5(0.2)$ & 0 & 0 & 0 & $32(1.5)$ \\
ASC-H & $34(1.6)$ & $9(0.4)$ & $1(0.04)$ & 0 & 0 & $44(2.1)$ \\
HSIL & $19(0.9)$ & $4(0.2)$ & 0 & 0 & 0 & $23(1.1)$ \\
SCC & $2(0.1)$ & $1(0.04)$ & 0 & 0 & 0 & $3(0.14)$ \\
AC & $1(0.04)$ & 0 & 0 & 0 & 0 & $1(0.04)$ \\
Total & $1656(78.0)$ & $424(20.2)$ & $17(0.8)$ & $1(0.04)$ & $1(0.04)$ & $2099(100)$ \\
(No/\%) & & & & & & \\
\hline
\end{tabular}

No: Number of patients; ASCUS: atypical squamous cells of undetermined significance; LSIL: low-grade squamous intraepithelial lesion; ASC-H: atypical squamous cells, cannot exclude HSIL; HSIL: high-grade squamous intraepithelial lesion; SCC: squamous cell carcinoma; AC: adenocarcinoma.

cording to ethnic group, Fisher's exact test was used. The test statistic value was 5.802 ( $p$-value $=0.468)$, so it cannot be said that there is a difference in test results according to ethnic group $(p>0.05)$.

\section{HPV genotyping test results}

Of the 2099 patients in the study population, 32\% (671/2099) had one or more positive HPV results. Among those who tested positive, $57.7 \%(387 / 671)$ were high-risk single infections, $15.8 \%$ (106/671) were combined high- and low-risk infections, $14.9 \%(100 / 671)$ were high-risk multiple infections, $9.9 \%(67 / 671)$ were low-risk single infections, and $1.6 \%(11 / 671)$ were low-risk multiple infections (Table 4). When the positive HPV results were assessed by age, the group with the highest infection rate was $30-39$ years with $31.6 \%(212 / 671)$, followed by $40-49$ years with $28.2 \%$ (189/671), 50-59 years with $18.9 \%$ (127/671), 20-29 years with $12.8 \%$ (86/671), 60-69 years with $7.0 \%$ (47/671), and over 70 years with $1.5 \%(10 / 671)$ (Table 
Table 4. - Overall correlation of cytology with human papilloma virus molecular result.

\begin{tabular}{|c|c|c|c|c|c|c|c|}
\hline \multirow{2}{*}{$\begin{array}{l}\text { Cytology } \\
\text { Diagnosis }\end{array}$} & \multirow{2}{*}{$\mathrm{No} / \%$} & \multirow{2}{*}{$\operatorname{HPV}(+)$} & \multicolumn{5}{|c|}{ HPV infection pattern } \\
\hline & & & $\mathrm{H}-\mathrm{S}$ & H-M & H\&L-M & L-S & L-M \\
\hline Total & $2099(100)$ & $671(32)$ & $387(18.4)$ & $100(4.7)$ & $106(5.1)$ & $67(3.2)$ & $11(0.5)$ \\
\hline Normal & $1783(85)$ & $419(20)$ & $253(12.1)$ & $49(2.3)$ & $58(2.8)$ & $52(2.5)$ & $7(0.3)$ \\
\hline Abnormal & $316(15)$ & $252(12)$ & $134(6.3)$ & $51(2.4)$ & $48(2.3)$ & $15(0.7)$ & $4(0.2)$ \\
\hline
\end{tabular}

No: Number of patients; H-S: High-risk single infection, H-M: High-risk multiple infection, H\&L-M: High-risk \& Low-risk infection, L-S: Low-risk single infection, L-M: Low-risk multiple infection.

Table 5. - Age distribution of human papilloma virus infection and cytological abnormality.

\begin{tabular}{|c|c|c|c|c|c|}
\hline \multirow{2}{*}{\multicolumn{2}{|c|}{ Cytology diagnosis }} & \multicolumn{4}{|c|}{ HPV fluorescence genotyping } \\
\hline & & \multicolumn{2}{|c|}{$\begin{array}{l}\text { Cytological abnormal } \\
\qquad(\mathrm{No}=316)\end{array}$} & \multicolumn{2}{|c|}{$\begin{array}{l}\text { Cytological normal } \\
\qquad(\mathrm{No}=1783)\end{array}$} \\
\hline Age & $(\mathrm{No} / \%)$ & HPV negative & HPV positive & HPV negative & HPV positive \\
\hline Total & $2099(100)$ & $64(3.0)$ & $252(11.9)$ & $1364(65.0)$ & $419(20.2)$ \\
\hline$<20$ & $3(0.1)$ & 0 & 0 & $3(0.1)$ & 0 \\
\hline $20-29$ & $213(10.1)$ & $8(0.4)$ & $32(1.5)$ & $119(5.7)$ & $54(2.6)$ \\
\hline $30-39$ & $658(31.3)$ & $12(0.6)$ & $76(3.6)$ & $434(20.7)$ & $136(6.5)$ \\
\hline $40-49$ & $697(33.2)$ & $19(0.9)$ & $64(3.0)$ & $489(23.3)$ & $125(6.0)$ \\
\hline $50-59$ & 409 (19.5) & $20(0.9)$ & $52(2.5)$ & $262(12.5)$ & $75(3.6)$ \\
\hline $60-69$ & $101(4.8)$ & $3(0.1)$ & $24(1.1)$ & $51(2.4)$ & $23(1.1)$ \\
\hline$\geq 70$ & $18(0.9)$ & $2(0.1)$ & $4(0.2)$ & $6(0.3)$ & $6(0.2)$ \\
\hline
\end{tabular}

No: Number of patients.

5). The HPV-positive rate in Korean-Chinese women was $34.4 \%(146 / 424)$, which is slightly higher than the overall positive rate of $32 \%(671 / 2099)$ and the rate of $31 \%$ (514/1656) in Han-Chinese women. The Myo- and JangChinese total positive rates were $100 \%(1 / 1)$ for each group and the Man-Chinese positive rate was $52.9 \%(9 / 17)$. There was only one Jang-Chinese woman and one Myo-Chinese woman, so statistically significant results could not be obtained for these groups (Table 6). The most common HPV genotypes found in the Korean-Chinese group were HPV $52,58,16,53$, and 33, which differed from those in the Han-Chinese group, where the most common were 16, 52, 58,53 and 81 (Table 7). As a result of cross-analysis of HPV types 16, 18, 6, and 11 in the Han-Chinese $(\mathrm{n}=151)$, Korean-Chinese $(n=28)$, and Man-Chinese $(n=3)$, cells with a frequency less than 5 are more than $20 \%$ of the total cells. Therefore, Fisher's exact test was used. The test statistic value was 9.603 ( $p$-value $=0.096$ ), so it cannot be said that there is a significant difference between the Chinese, Korean-Chinese, and Man-Chinese groups for HPV types $16,18,6$, and 11 at the 0.05 significance level.

\section{Comparison of cervical cytology with diagnostics and HPV molecular test results}

In 1783 patients $(85 \%)$ who were diagnosed as normal by the LBC test, 419 (20\%) patients were confirmed to have an HPV infection according to Pap smear cytology. Of these 671 HPV-positive samples, $23.5 \%$ were diagnosed as ASCUS (158/671), 5.5\% as ASC-H (37/671), 4.6\% as LSIL (31/671), 3.3\% as HSIL (22/671), $0.45 \%$ as SCC (3/671), and $0.15 \%$ as $\mathrm{AC}(1 / 671)$. Of the 316 patients who showed abnormalities according to the LBC cytological diagnosis, 64 (3.1\%) cases of HPV infection were not identified. When compared to the diagnoses by DNA testing, Pap smear cytology showed that $37.5 \%$ were true positives (252/671), 95.5\% were true negatives (1364/1428), 4.5\% were false positives $(64 / 1428)$, and $62.4 \%$ were false negatives $(419 / 671)$. The five most common genotypes in patients with abnormal cytological findings were HPV 16, 52, 58,56 , and 53, sequentially. The five most common genotypes in patients with normal cytological findings were sequentially HPV 52, 16, 58, 53, 81. Overall, the five most common HPV genotypes were sequentially $16,52,58,53$, 56 (Table 8-10).

\section{Discussion}

HPV is a DNA virus that is an important pathogen associated with cervical cancer. Persistent HPV infections, especially of high-risk genotypes, play an important role in the progression of progenitor lesions to invasive cancer [2]. The virus can be present in cells while the cytological transformation is not yet apparent, but the cytological test is still being performed as the screening test for cervical cancer. We show that the overall positive rate of HPV infection diagnosed by DNA testing was 32\% (671/2099) 
Table 6. - Ethnic difference of human papilloma virus prevalence and cytological abnormality.

\begin{tabular}{|c|c|c|c|c|c|}
\hline \multirow[b]{3}{*}{ Race } & \multirow{3}{*}{$\begin{array}{c}\text { gy diagnosis } \\
(\mathrm{No} / \%)\end{array}$} & \multicolumn{4}{|c|}{ HPV Genotyping } \\
\hline & & \multicolumn{2}{|c|}{$\begin{array}{l}\text { Cytological abnormal } \\
\qquad(\mathrm{n}=316)\end{array}$} & \multicolumn{2}{|c|}{$\begin{array}{l}\text { Cytological normal } \\
\qquad(\mathrm{n}=1783)\end{array}$} \\
\hline & & HPV negative & HPV positive & HPV negative & HPV positive \\
\hline Total & $2099(100)$ & $64(3.1)$ & $252(12.0)$ & $1364(65.0)$ & $419(20.0)$ \\
\hline Han-Chinese & $1656(78.9)$ & $47(2.2)$ & $195(9.3)$ & $1095(52.2)$ & $319(15.1)$ \\
\hline Korean- Chinese & $424(20.2)$ & $16(0.8)$ & $53(2.5)$ & $262(12.5)$ & $93(4.4)$ \\
\hline Man-Chinese & $17(0.8)$ & $1(0.04)$ & $3(0.1)$ & $7(0.3)$ & $6(0.3)$ \\
\hline Jang-Chinese & $1(0.04)$ & 0 & 0 & 0 & $1(0.04)$ \\
\hline Myo-Chinese & $1(0.04)$ & 0 & $1(0.04)$ & 0 & 0 \\
\hline
\end{tabular}

No: Number of patients.

Table 7. - Ethnic differences in human papilloma virus subtype distribution.

\begin{tabular}{|c|c|c|c|c|c|c|c|}
\hline & \multirow{2}{*}{ HPV type } & \multicolumn{5}{|c|}{ Ethnic classification (No/\%) } & \multirow{2}{*}{ Total $(\mathrm{No} / \%)$} \\
\hline & & Han-Chinese & Korean-Chinese & Man-Chinese & Jang-Chinese & Myo-Chinese & \\
\hline \multirow[t]{15}{*}{ High-cisk } & 16 & $112(11.0)$ & $17(1.6)$ & $2(0.2)$ & 0 & 0 & $131(12.8)$ \\
\hline & 18 & $15(1.4)$ & $6(0.6)$ & $1(0.1)$ & 0 & 0 & $22(2.1)$ \\
\hline & 31 & $31(3.0)$ & $8(0.8)$ & $2(0.2)$ & 0 & 0 & $41(4.0)$ \\
\hline & 33 & $25(2.4)$ & $14(1.3)$ & 0 & 0 & $1(0.1)$ & $40(3.8)$ \\
\hline & 35 & $14(1.3)$ & $4(0.4)$ & 0 & 0 & 0 & $18(1.7)$ \\
\hline & 39 & $27(2.6)$ & $10(1.0)$ & $1(0.1)$ & 0 & $1(0.1)$ & $39(3.8)$ \\
\hline & 45 & $7(0.7)$ & $4(0.4)$ & $1(0.1)$ & 0 & 0 & $12(1.2)$ \\
\hline & 51 & $38(3.7)$ & $12(1.2)$ & $1(0.1)$ & 0 & 0 & $51(5.0)$ \\
\hline & 52 & $82(7.9)$ & $43(4.1)$ & $1(0.1)$ & 0 & 0 & $126(12.1)$ \\
\hline & 53 & $54(5.2)$ & $16(1.5)$ & 0 & $1(0.1)$ & 0 & $71(6.8)$ \\
\hline & 56 & $39(3.8)$ & $13(1.3)$ & 0 & 0 & 0 & $52(5.1)$ \\
\hline & 58 & 79 (7.6) & $18(1.7)$ & 0 & 0 & $1(0.1)$ & $98(9.4)$ \\
\hline & 59 & $29(2.8)$ & $4(0.4)$ & 0 & 0 & 0 & $33(3.2)$ \\
\hline & 66 & $39(3.8)$ & $6(0.6)$ & 0 & 0 & 0 & $45(4.4)$ \\
\hline & 68 & $30(2.9)$ & $10(1.0)$ & 0 & 0 & 0 & $40(3.9)$ \\
\hline \multirow[t]{8}{*}{ Low-cisk } & 6 & $12(1.2)$ & $5(0.5)$ & 0 & 0 & 0 & $17(1.7)$ \\
\hline & 11 & $12(1.2)$ & 0 & 0 & 0 & 0 & $12(1.2)$ \\
\hline & 42 & $38(3.7)$ & $13(1.3)$ & 0 & 0 & 0 & $51(5.0)$ \\
\hline & 43 & $22(2.1)$ & $9(0.9)$ & 0 & 0 & 0 & $31(3.0)$ \\
\hline & 44 & $26(2.5)$ & $10(1.0)$ & 0 & 0 & 0 & $36(3.6)$ \\
\hline & 81 & $41(4.0)$ & $8(0.8)$ & $1(0.1)$ & 0 & 0 & $50(4.9)$ \\
\hline & 73 & $9(0.9)$ & $4(0.4)$ & 0 & 0 & 0 & $13(1.3)$ \\
\hline & 82 & $9(0.9)$ & $1(0.1)$ & 0 & 0 & 0 & $10(1.0)$ \\
\hline Total & & $790(76.0)$ & $235(22.6)$ & $10(1.0)$ & $1(0.1)$ & $3(0.3)$ & $1039(100)$ \\
\hline
\end{tabular}

No: Number of patients.

in Korean-Chinese women. This is higher than the global positive rate, which ranges from 1.4-25.6\% [18]. Abnormal cytological diagnosis (ASCUS, LSIL, HSIL, SCC, AC) was seen in $15.2 \%$ of the study population (316/2099), and high-risk single infection comprised 6.3\% (134/2099), which was higher than the rate of high-risk multiple infection at $2.4 \%(51 / 2099)$. In this study, HPV high-risk single infections were more frequently seen in HSIL and SCC than higher-grade multiple infections, which is in contrast to the fact that complex infections can cause persistent in- fections [27]. This result confirms the limitation of low sensitivity, although the cervical cytology technique used previously was successful in screening for cervical cancer. Therefore, the results of our study suggest that the HPV molecular test should be used alongside the LBC test. In this experiment, among the positive cases in the molecular test, $20 \%$ of cases showed cytological negativity. Results obtained from women in various regions of China and Korea were similarly shown to be $51.9 \%(41 / 79) \mathrm{HPV}$-positive in Shandong, China; 33.6\% HPV-positive in Seoul, Ko- 
Table 8. - Correlation of liquid-based cytology with human papilloma virus diagnosis.

\begin{tabular}{lccc}
\hline \multirow{2}{*}{ Cytology diagnosis } & \multicolumn{2}{c}{ HPV fluorescence Genotyping (No/\%) } & Total \\
\cline { 2 - 4 } & HPV - & HPV + & No/\%) \\
\hline Normal & $1364(65)$ & $419(20)$ & $1783(85)$ \\
ASCUS & $55(2.6)$ & $158(7.5)$ & $213(10.1)$ \\
LSIL & $1(0.04)$ & $31(1.5)$ & $32(1.5)$ \\
ASC-H & $7(0.3)$ & $37(1.8)$ & $44(2.1)$ \\
HSIL & $1(0.04)$ & $22(1.04)$ & $23(1.1)$ \\
SCC & & $3(0.14)$ & $3(0.14)$ \\
AC & & $1(0.04)$ & $1(0.04)$ \\
Total (No/\%) & $1428(68)$ & $671(32)$ & $2099(100)$ \\
\hline
\end{tabular}

No: Number of patients; ASCUS: atypical squamous cells of undetermined significance; LSIL: low-grade squamous intraepithelial lesion; ASC-H: atypical squamous cells, cannot exclude HSIL; HSIL: high-grade squamous intraepithelial lesion; SCC: squamous cell carcinoma; AC: adenocarcinoma.

Table 9. - Correlation of human papilloma virus genotype with cytological result.

\begin{tabular}{|c|c|c|c|c|c|c|c|c|c|c|}
\hline & \multirow{2}{*}{ HPV type } & \multirow{2}{*}{$\begin{array}{l}\text { Normal cytology } \\
\qquad(\mathrm{No} / \%)\end{array}$} & \multicolumn{2}{|c|}{ Abnormal cytology } & \multicolumn{4}{|c|}{ Abnormal cytology $(\mathrm{No} / \%)$} & \multicolumn{2}{|r|}{ Total $(\mathrm{No} / \%)$} \\
\hline & & & $(\mathrm{No} / \%)$ & ASCUS & ASC-H & LSIL & HSIL & SCC & $\mathrm{AC}$ & \\
\hline \multirow{15}{*}{$\begin{array}{l}\text { High- } \\
\text { risk }\end{array}$} & 16 & $71(6.8)$ & $60(5.8)$ & $20(2.0)$ & $18(1.7)$ & $5(0.5)$ & $13(1.2)$ & $3(0.3)$ & $1(0.1)$ & $131(12.6)$ \\
\hline & 18 & $16(1.5)$ & $6(0.6)$ & $4(0.4)$ & & $1(0.1)$ & $1(0.1)$ & & & $22(2.1)$ \\
\hline & 31 & $24(2.3)$ & $17(1.6)$ & $8(0.8)$ & & $6(0.6)$ & $3(0.3)$ & & & $41(3.9)$ \\
\hline & 33 & $19(1.8)$ & $21(2.0)$ & $14(1.3)$ & $3(0.3)$ & $1(0.1)$ & $3(0.3)$ & & & $40(3.8)$ \\
\hline & 35 & $10(0.1)$ & $8(0.8)$ & $6(0.6)$ & $1(0.1)$ & $1(0.1)$ & & & & $18(1.7)$ \\
\hline & 39 & $18(1.7)$ & $21(2.0)$ & $18(1.7)$ & $1(0.1)$ & $2(0.2)$ & & & & $39(3.8)$ \\
\hline & 45 & $11(1.1)$ & $1(0.1)$ & & $1(0.1)$ & & & & & $12(1.2)$ \\
\hline & 51 & $27(2.6)$ & $24(2.3)$ & $14(1.3)$ & $6(0.6)$ & $2(0.2)$ & $2(0.2)$ & & & $51(4.9)$ \\
\hline & 52 & $77(7.4)$ & $49(4.7)$ & $35(3.4)$ & $8(0.8)$ & $4(0.4)$ & $2(0.2)$ & & & $126(12.1)$ \\
\hline & 53 & $46(4.4)$ & $25(2.4)$ & $15(1.4)$ & & $7(0.7)$ & $2(0.2)$ & & $1(0.1)$ & $71(6.8)$ \\
\hline & 56 & $23(2.2)$ & $29(2.8)$ & $21(2.0)$ & & $6(0.6)$ & $2(0.2)$ & & & $52(5.0)$ \\
\hline & 58 & $54(5.2)$ & $44(4.2)$ & $30(2.9)$ & $6(0.6)$ & $4(0.4)$ & $4(0.4)$ & & & $98(9.4)$ \\
\hline & 59 & $25(2.5)$ & $8(0.8)$ & $5(0.5)$ & $1(0.1)$ & $2(0.2)$ & & & & $33(3.2)$ \\
\hline & 66 & $23(2.2)$ & $22(2.1)$ & $15(1.4)$ & $1(0.1)$ & $5(0.5)$ & $1(0.1)$ & & & $45(4.3)$ \\
\hline & 68 & $23(2.2)$ & 17 (1.6) & $13(1.3)$ & $1(0.1)$ & $2(0.2)$ & $1(0.1)$ & & & $40(3.8)$ \\
\hline \multirow{8}{*}{$\begin{array}{l}\text { Low- } \\
\text { risk }\end{array}$} & 6 & $9(0.9)$ & $8(0.8)$ & $4(0.4)$ & & $2(0.2)$ & $1(0.1)$ & & $1(0.1)$ & $17(1.6)$ \\
\hline & 11 & $4(0.4)$ & $8(0.8)$ & $7(0.7)$ & $1(0.1)$ & & & & & $12(1.2)$ \\
\hline & 42 & $30(2.9)$ & $21(2.0)$ & $12(1.2)$ & $3(0.3)$ & $4(0.4)$ & $1(0.1)$ & & $1(0.1)$ & $51(4.9)$ \\
\hline & 43 & $25(2.4)$ & $6(0.6)$ & $5(0.5)$ & & $1(0.1)$ & & & & $31(3.0)$ \\
\hline & 44 & $23(2.2)$ & $13(1.3)$ & $10(1.0)$ & $1(0.1)$ & $2(0.2)$ & & & & $36(3.5)$ \\
\hline & 81 & $35(3.4)$ & $15(1.4)$ & $11(1.1)$ & $1(0.1)$ & $3(0.3)$ & & & & $50(4.8)$ \\
\hline & 73 & $8(0.8)$ & $5(0.5)$ & $2(0.2)$ & & $2(0.2)$ & $1(0.1)$ & & & $13(1.3)$ \\
\hline & 82 & $6(0.6)$ & $4(0.4)$ & $1(0.1)$ & $1(0.1)$ & $2(0.2)$ & & & & $10(1.0)$ \\
\hline Total & & $607(58.4)$ & $432(41.6)$ & $270(26.1)$ & $54(5.2)$ & $64(6.2)$ & $37(3.6)$ & $3(0.3)$ & $4(0.4)$ & $1039(100)$ \\
\hline
\end{tabular}

No: Number of patients; ASCUS: atypical squamous cells of undetermined significance; LSIL: low-grade squamous intraepithelial lesion; ASC-H: atypical squamous cells, cannot exclude HSIL; HSIL: high-grade squamous intraepithelial lesion; SCC: squamous cell carcinoma; AC: adenocarcinoma.

rea; 33.9\% (116/180) HPV-positive in Ghangwon, Korea; 8.5\% (70/821) HPV-positive in Busan, Korea; and 15.8\% (309/1960) HPV-positive in Daejeon and Daegu, Korea [23, 28-31]. Moreover, the high false-negative rate (62.4\%) of
LBC clearly shows the importance of combining the HPV genotyping test with LBC. Our data indicate that the cervical cancer screening system in Yanbian, China also requires a combination of cytological and HPV molecular 
Table 10. - Predictive value of liquid-based cytology examination.

\begin{tabular}{|c|c|c|c|c|}
\hline & \multicolumn{3}{|c|}{ HPV molecular test result No (\%) } & \multirow[t]{2}{*}{ Total } \\
\hline & & Positive & Negative & \\
\hline \multirow{2}{*}{ Cytology Result } & Positive & $252(12)$ & $64(3)$ & $316(15)$ \\
\hline & Negative & $419(20)$ & $1364(65)$ & $1783(85)$ \\
\hline Total & & $671(32)$ & $1428(68)$ & $2099(100)$ \\
\hline
\end{tabular}

No: Number of patients.

tests. In 2012, the American Cancer Society Screening Guidelines recommended that a combination of cytology and HPV DNA testing is the first choice for the prevention and early detection of cervical cancer [32].

In this study, the HPV-positive rate of Korean-Chinese women was shown to be $34.4 \%(146 / 424)$, which is slightly higher than the $31 \%(514 / 1656)$ in Han-Chinese women. This is about three times higher than the HPV-positive rate of $10.3 \%$ (90/870) in Busan, Korea [28], and higher than the overall positive rate of $26.6 \%(629 / 2368)$ in Daejeon and Daegu, Korea [23]. These reported results show HPV epidemiological diversity in infection rate and genotype by country, geography, race, genetic factors, and immune status. The diversity in infection rates can be explained in terms of vaccines or hygiene. Women in Yanbian, China carrying one or more HPV infections accounted for 32.3\% of the study population. This result is similar to the $31 \%$ of combined infections in US women [33] but much higher than the $19 \%$ in Korean women in Busan [28]. Age-based analyses can predict the onset of cervical cancer in high-risk groups and can help to determine the best age for focused screening projects [34]. In this study, the HPV-positive rate of Korean-Chinese women was the highest among those aged 30-39 years, followed by 40-49 years, 50-59 years, 20-29 years, and 60 years old. The age groups with the highest HPV-positive rates for Han-Chinese women were the 40s, 50s, 30s, under 30, and 60s [34], while for Mexican women, it was the $20 \mathrm{~s}, 30 \mathrm{~s}, 60 \mathrm{~s}, 50 \mathrm{~s}$, and $40 \mathrm{~s}$ [35]. In the US, the HPV prevalence is approximately $40 \%$ for women in the 20-29-year age group, with a gradual decline in older women [36].

HPV has 120 genetic subtypes, of which 16 (HPV 16, 18, $31,33,35,39,45,51,52,53,54,56,58,59,66$, and 68) have been described as high-risk. In particular, HPV 16 is known to be the world's most prevalent high-risk genotype. The next most common is HPV 18, but HPV 58 is often reported in Asia [19]. Among women living in the Yanbian Autonomous Region of Jilin Province in China, the five most common HPV genotypes were 16, 52, 58, 53, and 56. Within this population, the most prevalent HPV genotypes in Korean-Chinese women were 52, 58, 16, 53, and 33 (in descending order), whereas in the Han-Chinese women, the HPV genotypes were $16,52,58,53$, and 81 . For comparison, women living in Seoul have been diagnosed with HPV genotypes 53, 52, 58, 16, and 68 [30], those in the Gangwon region with genotypes $16,53,58,56$, and 70 [31], those in the Busan region with genotypes 70, 16, and 33 [28], and those in Taiwan and Japan with genotypes 52, 16, 51, 35, and 18 [18]. In China, the most prevalent HPV genotypes among the Shandong Han-Chinese were 16, 53, 18, 58, and 33 [29], while in 37 Chinese cities, the HPV genotypes 16, $52,58,59$, and 39 were the most common [37]. The difference in subtypes of HPV infection by region or race can be explained in connection with the degree of openness of sexual partners. These data, together with our own, show that the HPV genotypes in infected women differed by race and region, and indicate that the choice of vaccine should therefore depend on the genotype of HPV infections in that area.

There are two types of HPV vaccine, Cervarix and Gardasil [25]. The Cervarix 2-valent vaccine is a suspension for intramuscular injection containing purified viral L1 protein for HPV 16 and 18. It is available in 1- or 2-dose vials or prefilled syringes, and is produced using a baculovirus expression system in Trichoplusia ni cells. Gardasil is produced as either a 4-valent or 9-valent HPV vaccine. The 4valent $\mathrm{HPV}$ vaccine is a suspension for intramuscular injection containing purified viral L1 protein for HPV 6, 11, 16, and 18 , and is available in 1-dose vials or prefilled syringes. The vaccine is produced using a yeast substrate and includes amorphous aluminum hydroxyphosphate sulfate as an adjuvant. The 9-valent HPV vaccine is a suspension for intramuscular injection containing purified viral L1 protein for 9 HPV genotypes $(6,11,16,18,31,33,45,52$, and 58) [25, $26]$. Given that the results of our study show that HPV 52, $58,16,53$, and 33 were the most highly prevalent subtypes in Korean-Chinese women living in Yanbian, it is anticipated that the Gardasil 9-valent HPV vaccine may be more useful in the prevention of cervical lesions in these women.

In summary, the HPV infection status of KoreanChinese women living in the Yanbian Korean Autonomous Region of Jilin Province in China differed from that of other races and regions in terms of infection rate and genotype. In addition, screening for HPV by LBC alone did not effectively diagnose HPV infection. The current study has yielded epidemiological data on the prevalence and distribution of HPV in this area, which may be used in selecting appropriate vaccines and designing prevention plans. More research about why the genotype of HPV among Korean-Chinese women differs from the Koreans or HanChinese women in the same region is needed. The results of this study will be an important contribution toward vaccine 
planning and cervical cancer prevention projects in Jilin Province, China.

\section{Ethics approval and consent to participate}

All subjects gave their informed consent for inclusion before they participated in the study. The study was conducted in accordance with the Declaration of Helsinki, and the protocol was approved by the Ethics Committee of Kangwon National University (approval number: 2019-12008-003).”

\section{Acknowledgments}

This study was supported by a 2017 Research Grant from Kangwon National University (No. 520170427).

\section{Conflict of Interest}

The authors report no conflicts of interest in this work.

Submitted: March 20, 2020

Accepted: May 13, 2020

Published: October 15, 2020

\section{References}

[1] Torre L.A., Bray F., Siegel R.L., Ferlay J., Lortet-Tieulent J., Jemal A.: "Global cancer statistics, 2012". Ca. Cancer J. Clin., 2015, 65, $87-108$.

[2] Walboomers J.M.M., Jacobs M.V., Manos M.M., Bosch F.X., Kummer J.A., Shah K.V., et al.: "Human papillomavirus is a necessary cause of invasive cervical cancer worldwide". the Journal of Pathology, 1999, 189, 12-19.

[3] Woodman C.B., Collins S., Winter H., Bailey A., Ellis J., Prior P., et al.: "Natural history of cervical human papillomavirus infection in young women: a longitudinal cohort study". the Lancet, 2001, 357, 1831-1836.

[4] Castle P.E., Wacholder S., Sherman M.E., Lorincz A.T., Glass A.G., Scott D.R., et al.: "Absolute risk of a subsequent abnormal pap among oncogenic human papillomavirus DNA-positive, cytologically negative women". Cancer, 2002, 95, 2145-2151.

[5] Rozendaal L., Walboomers J.M.M., van der Linden J.C., Voorhorst F.J., Kenemans P., Helmerhosrt T.J.M., et al.: "PCR-based highrisk HPV test in cervical cancer screening gives objective risk assessment of women with cytomorphologically normal cervical smears". Int. J. Cancer, 1996, 68, 766-769.

[6] Ylitalo N., Sørensen P., Josefsson A. M., Magnusson P. K., Andersen P. K., Pontén J., et al.: "Consistent high viral load of human papillomavirus 16 and risk of cervical carcinoma in situ: a nested case-control study". the Lancet, 2000, 355, 2194-2198.

[7] Naucler P., Ryd W., Törnberg S., Strand A., Wadell G., Hansson B. G., Rylander E., Dillner J.: "HPV type-specific risks of high-grade CIN during 4 years of follow-up: a population-based prospective study". Br. J. Cancer, 2007, 97, 129-132.

[8] Schiffman M., Clifford G., Buonaguro F.M.: "Classification of weakly carcinogenic human papillomavirus types: addressing the limits of epidemiology at the borderline". Infect. Agent. Cancer, 2009,4 ,

[9] Hwang T.S., Jeong J.K., Park M., Han H.S., Choi H.K., Park T.S.: "Detection and typing of HPV genotypes in various cervical lesions by HPV oligonucleotide microarray". Gynecol. Oncol., 2003, 90, 51-56.

[10] Muñoz N., Bosch F.X., de Sanjosé S., Herrero R., Castellsagué X., Shah K. V., et al:: "Epidemiologic Classification of Human Papillomavirus Types Associated with Cervical Cancer". N. Engl. J. Med., 2003, 348, 518-527.

[11] Agarossi A., Ferrazzi E., Parazzini F., Perno C.F., Ghisoni L.: "Prevalence and type distribution of high-risk human papillomavirus infection in women undergoing voluntary cervical cancer screening in Italy". J. Med. Virol., 2009, 81, 529-535.
[12] Wells S.I., Aronow B.J., Wise T.M., Williams S.S., Couget J.A., Howley P.M.: "Transcriptome signature of irreversible senescence in human papillomavirus-positive cervical cancer cells". Proceedings of the National Academy of Sciences, 2003, 100, 7093-7098.

[13] Hutchinson D.J., Klein K. C.: "Human papillomavirus disease and vaccines". am. J. Health. Syst. Pharm., 2008, 65, 2105-2112.

[14] de Sanjosé S., Brotons M., Pavón M.A.: "The natural history of human papillomavirus infection”. Best Practice \& Research Clinical Obstetrics \& Gynaecology, 2018, 47, 2-13.

[15] Berchtold A., Michaud P., Nardelli-Haefliger D., Surís J.: "Vaccination against human papillomavirus in Switzerland: simulation of the impact on infection rates". Int. J. Public Health, 2010, 55, 25-34.

[16] Cento V., Ciccozzi M., Ronga L., Perno C.F., Ciotti M.: "Genetic diversity of human papillomavirus type $16 \mathrm{E} 6, \mathrm{E} 7$, and L1 genes in Italian women with different grades of cervical lesions". J. Med. Virol., 2009, 81, 1627-1634.

[17] Rambout L., Hopkins L., Hutton B., Fergusson D.: "Prophylactic vaccination against human papillomavirus infection and disease in women: a systematic review of randomized controlled trials". can. Med. Assoc. J., 2007, 177, 469-479.

[18] de Sanjosé S., Diaz M., Castellsagué X., Clifford G., Bruni L., Muñoz N., et al.: "Worldwide prevalence and genotype distribution of cervical human papillomavirus DNA in women with normal cytology: a meta-analysis". the Lancet Infectious Diseases, 2007, 7, 453-459.

[19] Clifford G.M., Smith J.S., Plummer M., Muñoz N., Franceschi S.: "Human papillomavirus types in invasive cervical cancer worldwide: a meta-analysis". Br. J. Cancer, 2003, 88, 63-73.

[20] Senapati R., Nayak B., Kar S. K., Dwibedi B.: "HPV genotypes co-infections associated with cervical carcinoma: Special focus on phylogenetically related and non-vaccine targeted genotypes". Plos one, 2017, 12, $\mathrm{e} 0187844$.

[21] Zhang Y., Wang Y., Liu L., Fan Y., Liu Z., Wang Y., et al.: "Awareness and knowledge about human papillomavirus vaccination and its acceptance in China: a meta-analysis of 58 observational studies". Bmc Public Health, 2016, 16,

[22] Laudadio J.: "Human Papillomavirus Detection". Adv. Anat. Pathol., 2013, 20, 158-167.

[23] Hong S.R., Kim I.S., Kim D.W., Kim M.J., Kim A.R., Kim Y.O., et al:: "Prevalence and Genotype Distribution of Cervical Human Papillomavirus DNA in Korean Women: A Multicenter Study". the Korean Journal of Pathology, 2009, 43, 342.

[24] Oyervides-Muñoz M.A., Pérez-Maya A.A., Sánchez-Domínguez C.N., Berlanga-Garza A., Antonio-Macedo M., Valdéz-Chapa L.D., et al.: "Multiple HPV Infections and Viral Load Association in Persistent Cervical Lesions in Mexican Women”. Viruses, 2020, 12, 380.

[25] "Human papillomavirus vaccines: WHO position paper, May 2017". Wkly. Epidemiol. Rec., 2017, 92, 241-68.

[26] Kim M., No J.H., Song Y.: "Human Papillomavirus Vaccine". Journal of the Korean Medical Association, 2009, 52, 1180.

[27] Ho G.Y.F., Burk R.D., Klein S., Kadish A.S., Chang C.J., Palan P., et al.: "Persistent Genital Human Papillomavirus Infection as a Risk Factor for Persistent Cervical Dysplasia". JNCI Journal of the National Cancer Institute, 1995, 87, 1365-1371.

[28] Hong S.H., Lee D.H., Shin H.R.: "Prevalence of Human papillomavirus infection in women in South Korea: Incidence of positive HPV DNA and anti-VLPs in residents of Busan city". Korean. $J$. Cytopathol., 2004, 15, 17-27.

[29] Wang H.Y., Lee D., Park S., Kim G., Kim S., Han L., et al.: "Diagnostic performance of HPV E6/E7 mRNA and HPV DNA Assays for the Detection and Screening of Oncogenic Human Papillomavirus Infection among Woman with Cervical Lesions in China". Asian. Pac. J. Cancer. Prev., 2015, 16, 7633-7640.

[30] So K.A., Hong J.H., Lee J.K.: "Human Papillomavirus Prevalence and Type Distribution Among 968 Women in South Korea". Journal of Cancer Prevention, 2016, 21, 104-109.

[31] Lee D., Department of Pathology, Yonsei University Wonju College of Medicine, Wonju, Korea., Kim S., Park S., Jin H., Kim T. U., et al.: "Human Papillomavirus Prevalence in Gangwon Province Using Reverse Blot Hybridization Assay". the Korean Journal of Pathology, 2011, 45, 348.

[32] Saslow D., Solomon D., Lawson H.W., Killackey M., Kulasingam S.L., Cain J., et al.: "American Society for Colposcopy and Cervical Pathology, and American Society for Clinical Pathology screening 
guidelines for the prevention and early detection of cervical cancer". Am. J. Clin. Pathol., 2012, 137, 516.

[33] Kulasingam S.L., Hughes J.P., Kiviat N.B., Mao C., Weiss N.S., Kuypers J. M., et al.: "Evaluation of Human Papillomavirus Testing in Primary Screening for Cervical Abnormalities". Jama, 2002, 288 , 1749.

[34] Kim G., Kim S., Park S., Park S., Lin H., Ren Y., et al.: "Human Papillomavirus Prevalence and Distribution according to Age among Korean and Chinese Women". Korean Journal of Clinical Laboratory Science, 2015, 47, 259-266.

[35] Magaña-Contreras M., Contreras-Paredes A., Chavez-Blanco A., Lizano M., Cruz-Hernandez Y.D.L., Cruz-Hernandez E.D.L.: "Prevalence of sexually transmitted pathogens associated with HPV infection in cervical samples in a Mexican population". J. Med. Virol., 2015, 87, 2098-2105.

[36] Stoler M.H.: "Advances in Cervical Screening Technology". Mod.
Pathol., 2000, 13, 275-284.

[37] Wang R., Guo X., Wisman G.B.A., Schuuring E., Wang W., Zeng Z., et al:: "Nationwide prevalence of human papillomavirus infection and viral genotype distribution in 37 cities in China". Bmc Infect. Dis., 2015, 15.

Corresponding Author:

YOUNG-JOON RYU, M.D, Ph.D.

Department of Pathology, College of Medicine, Kangwon National University, Chun-Cheon, 200-701 (Korea)

e-mail: mindmader@gmail.com 